\title{
Influence of cross correlations on interdiffusion in Al-rich Al-Ni melts
}

\author{
Elke Sondermann, ${ }^{*}$ Florian Kargl, and Andreas Meyer \\ Institut für Materialphysik im Weltraum, Deutsches Zentrum für Luft- und Raumfahrt (DLR), 51170 Köln, Germany
}

(Received 10 August 2015; published 6 May 2016)

\begin{abstract}
The relation of self- and interdiffusion in a liquid metal, particularly the influence of cross correlations at low concentrations, is studied experimentally. Accurate interdiffusion data are obtained by a combination of x-ray radiography with the shear-cell method on the ground and on the sounding rocket MAPHEUS under microgravity conditions. Self-diffusion coefficients, measured by quasielastic neutron scattering, increase with decreasing Ni concentration, whereas interdiffusion coefficients are about constant. We show that cross correlations influence interdiffusion even at concentrations as low as 2 at. \% Ni. Consequently, Darken's equation is not valid in this case.
\end{abstract}

DOI: 10.1103/PhysRevB.93.184201

\section{INTRODUCTION}

Diffusion of mass is a fundamental property, which influences the velocity of chemical reactions, doping of semiconductors, sintering, and other processes [1]. In the case of liquid alloys, diffusion has an impact on the formation and evolution of microstructure during solidification [2]. Here, the redistribution of solute by diffusion through the liquid during dendrite growth is an essential process. The resulting microstructure determines properties of the solid, such as ductility or corrosion resistance. Much effort has been made to understand and predict the solidification process, including crystal growth and vitrification [3,4].

Along with front tracking models, phase-field modeling has become a widely used method to study solidification [5]. Different models in combination with an experimental database can be used to predict, e.g., the evolution of microsegregation during solidification or the growth kinetics of precipitates in multicomponent alloys [6]. For all of these simulations, accurate diffusion coefficients are needed. Changing the diffusion coefficient by a factor of 2 or neglecting its concentration and temperature dependences leads to a qualitative change of the simulated microstructure [2,7]. Cast aluminum alloys contain low concentrations of different alloying elements. Using experimental data, we investigate whether at low concentrations interdiffusion coefficients can be derived directly from self-diffusion coefficients, or whether cross correlations still impact interdiffusion.

Diffusion coefficients in liquid metals are often measured by the long-capillary method and its variations [8,9], where two rods with different concentrations of an alloy or with different amounts of an isotope are placed face to face in a capillary, melted, and kept at the desired temperature for a certain time. The diffusion coefficient can then be derived from the concentration profile of the solidified sample. Long capillaries are used in order to suppress convective contributions to mass transport. Nevertheless, measured diffusion data scatter by $10 \%$ to several $100 \%$ [10-13]. In addition to effects due to melting and solidification, buoyancy-driven convection and Marangoni convection, which is provoked by differences in surface tension, are the main sources of error [8,14,15].

\footnotetext{
*elke.sondermann@dlr.de
}

Recently, new techniques were developed to increase accuracy. To determine interdiffusion coefficients, x-ray radiography (XRR) is used to conduct time-resolved in situ measurements with improved process control [10,16,17]. Since the concentration profile is determined in the liquid state, solidification of the sample has no influence. Furthermore, free surfaces, which cause Marangoni convection, can be detected, and the respective measurements can be excluded from analysis. We combine XRR with the shear-cell method, where samples with different concentrations are melted separately and only brought together after temperature and concentration distribution are homogenized. This prevents segregation and mixing of alloys during heating. To suppress buoyancy-driven convection, such measurements were also conducted on the sounding rocket MAPHEUS-4 at a microgravity level better than $10^{-4} \mathrm{~g}$ [18].

Additional mass transport can be made visible through the time- and space-resolved information obtained by XRR. As an example, Fig. 1 shows the shear-induced convection that occurred only under microgravity. This convection is viscously damped within about $5 \mathrm{~s}$, in agreement with numerical calculations [19,20]. After about $30 \mathrm{~s}$, the concentration distribution approaches parallel concentration lines. By analyzing only undisturbed data, benchmark values for interdiffusion in liquid Al-Ni were obtained. XRR is restricted to materials with sufficient X-ray contrast.

Self-diffusion coefficients were measured by quasielastic neutron scattering (QENS) [21]. This method uses the fact that the elastic signal of the incoherent intermediate scattering function is broadened by the diffusive motion in liquids. Such a measurement probes diffusion on a ps time scale and is therefore undisturbed by convection $[22,23]$. To measure self-diffusion in alloys, the incoherent intermediate scattering function should be dominated by one component.

Al-rich Al-Ni alloys exhibit strong x-ray contrast. Furthermore, the incoherent signal in QENS is dominated by nickel [24]. In this system, accurate measurements of $\mathrm{Ni}$ self-diffusion and of interdiffusion are therefore possible. It can be used to study the relation between these two types of diffusion. Interdiffusion describes the collective transport of mass driven by differences in the chemical potential, e.g., due to concentration gradients in alloys, whereas self-diffusion is related to the mean-square displacement of tagged atoms. In a 

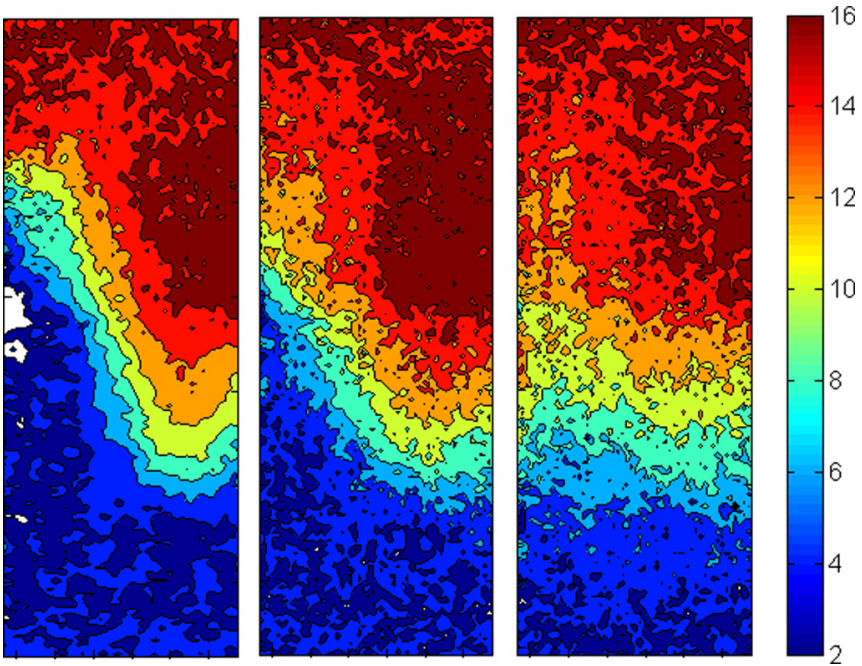

FIG. 1. Two-dimensional projection of the concentration distribution of the diffusion couple Al-Ni 4 at. \%-Al-Ni 16 at. \%. Images are obtained under microgravity 1,5 , and $30 \mathrm{~s}$ after starting the diffusion process through a shear movement. The dimension of each cutout is $3.5 \times 1.2 \mathrm{~mm}$. The color scale indicates the Ni concentration in at. \%. A clear shear-induced convection can be seen, but after $30 \mathrm{~s}$ the concentration distribution approaches parallel concentration lines.

binary alloy with concentrations $c_{\mathrm{Al}}$ and $c_{\mathrm{Ni}}$, the interdiffusion coefficient $D_{\mathrm{AlNi}}$ is connected to the self-diffusion coefficients $D_{\mathrm{Al}}$ and $D_{\mathrm{Ni}}$ by the thermodynamic factor $\Phi$ and a factor $S$ that measures the contribution of cross correlations to interdiffusion [25]:

$$
D_{\mathrm{AlNi}}=S \Phi\left(c_{\mathrm{Ni}} D_{\mathrm{Al}}+c_{\mathrm{Al}} D_{\mathrm{Ni}}\right) .
$$

For $S=1$ this is known as Darken's equation [26], which can be used to estimate missing diffusion coefficients.

Computer simulations of Al-Ni show $S<1$ for Ni content between 20 and 90 at. \% [24,27] over a wide temperature range. The impact of cross correlations is largest for concentrations around 60 at. $\% \mathrm{Ni}$ and is expected to vanish as $c_{\mathrm{Ni}} \rightarrow 0$. Cross correlations influence interdiffusion, e.g., if chemical shortrange order is present [27]. In addition, mass asymmetry of the particles increases the contribution of cross correlation [28]. In general, Darken's equation assumes a common relaxation velocity for all components [29].

\section{METHODS}

Samples used for the experiments were prepared from the pure elements (Al: granules $99.99 \% \mathrm{Cerac}, \mathrm{Ni}$ : slugs 99.995\% Alfa Aesar) by arc melting under a purified argon atmosphere. The shear cell (SC) consists of a graphite crucible for samples with a length of $15 \mathrm{~mm}$ and a diameter of 1.5 or $1.0 \mathrm{~mm}$. It is heated by electric resistance heaters made of graphite foil, which are electrically isolated by boron nitride plates. Special care has been taken regarding temperature homogeneity. Technical details of the setup can be found in $[30,31]$.

A microfocus x-ray source (Viscom) with a tungsten target, operated at $100 \mathrm{kV}$ with $15 \mathrm{~W}$, and equipped with a W-Cu aperture is used in combination with the detector Shad-o-Box
2048 (Rad-icon) at a frame rate of $0.5 \mathrm{~Hz}$. The effective pixel size is $25 \mu \mathrm{m}$. The obtained gray value images are converted into concentration profiles using the concentrations at the end of the capillary as references. The linear relation between $\mathrm{Ni}$ content and extinction of $\mathrm{x}$-ray radiation was verified for this setup. Gray value profiles were corrected for changes in detector or x-ray source performance and nonuniformity in capillary diameter when necessary. The obtained concentration profiles were fitted using the solution of the diffusion equation for two semi-infinite media with the starting concentrations $c_{1}$ and $c_{2}$, the center of the diffusion profile at $x_{0}$, and the error function erf [32]:

$$
c(t, x)=\frac{c_{1}+c_{2}}{2}+\frac{c_{2}-c_{1}}{2} \operatorname{erf}\left(\frac{x-x_{0}}{\sqrt{4 D_{\mathrm{AlNi}} t}}\right) .
$$

To detect possible convection rolls and to estimate the uncertainty of each measurement, the left, center, and right part of each capillary were analyzed separately [10]. Convection rolls in the beam direction cannot be detected. The influence of convection increases with capillary diameter [33,34]. For this reason, experiments were realized with sample diameters of 1.0 and $1.5 \mathrm{~mm}$, leading to the same diffusion coefficient. It was further verified that the concentration difference between the two parts of a diffusion couple does not alter the measured value, as can be seen in Fig. 2.

Neutron-scattering experiments were conducted at the timeof-flight spectrometer TOFTOF at the neutron source FRM II in Garching, near Munich. Samples with 2 and 5 at. \% Ni were processed in a cylindrical $\mathrm{Al}_{2} \mathrm{O}_{3}$ crucible at temperatures between 973 and $1350 \mathrm{~K}$. The incident neutron wavelength was $7 \AA$. The self-diffusion coefficient $D_{\mathrm{Ni}}$ is derived from the full width at half-maximum of the quasielastic line $\Gamma$ at small wave numbers $q$ via $D_{\mathrm{Ni}}=\Gamma /\left(2 \hbar q^{2}\right)$. The data analysis follows the procedure described in $[22,24]$.

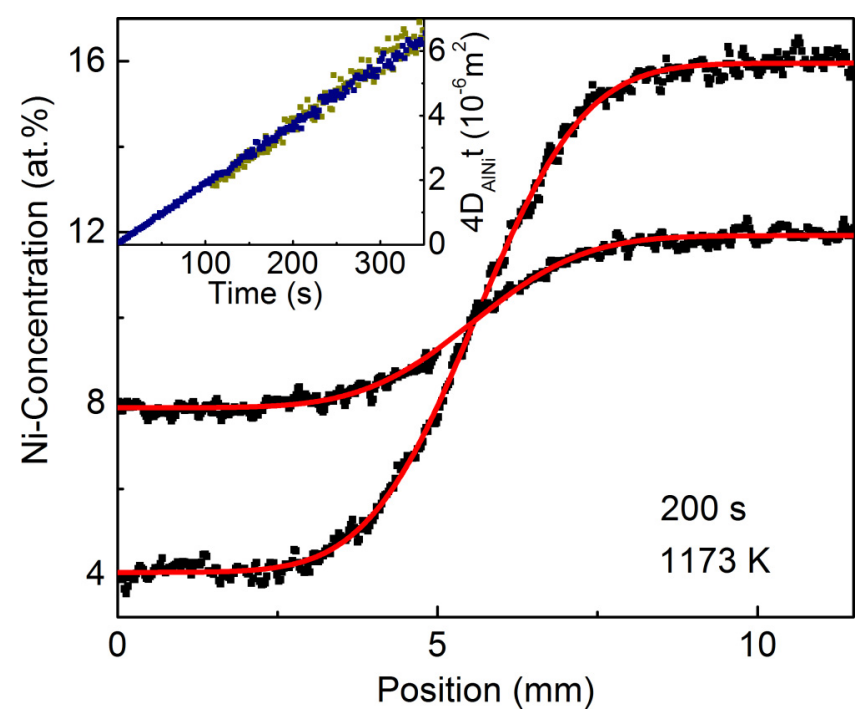

FIG. 2. Concentration profile of two experiments with a different spread around the mean concentration $c_{\mathrm{Ni}}=10$ at. $\% 200 \mathrm{~s}$ after the start of diffusion. Inset: $4 D_{\mathrm{AlNi}} t$ from the fit with Eq. (2) for these two experiments showing a linear time dependence with coinciding slope. 


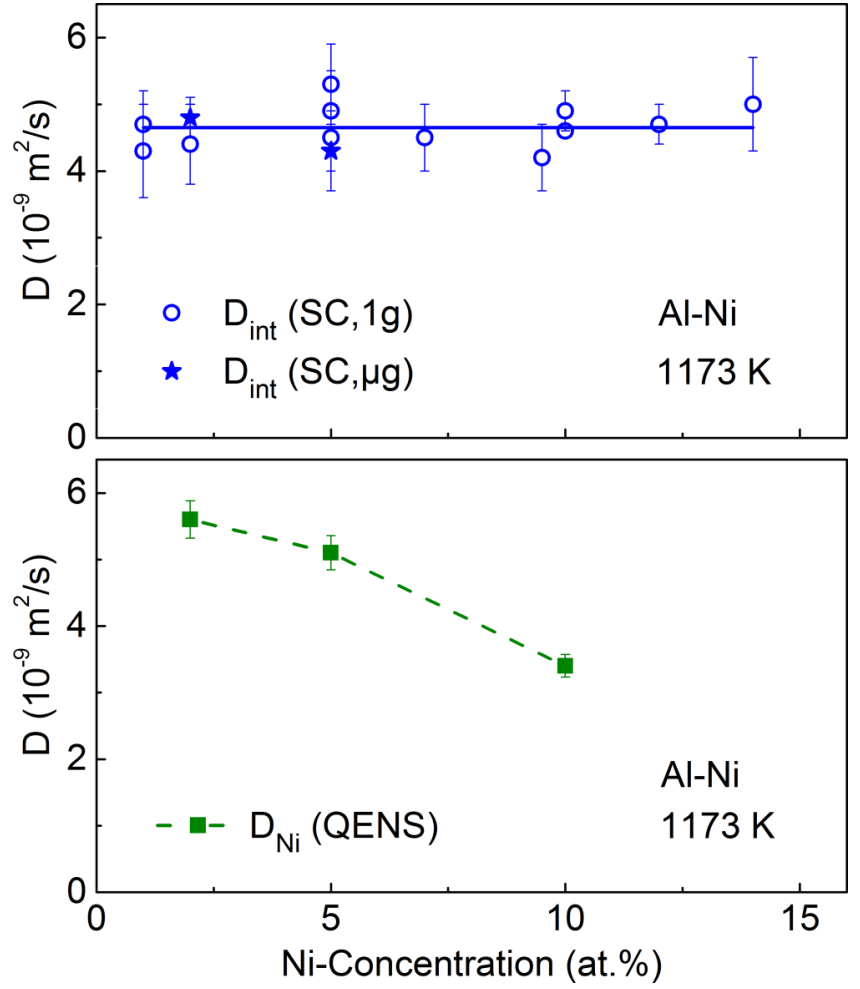

FIG. 3. Top: Interdiffusion coefficients with mean value shown as a solid line as a function of Ni concentration. Bottom: Self-diffusion coefficients for nickel as a function of $\mathrm{Ni}$ concentration. $D_{\mathrm{Ni}}$ at 10 at. $\% \mathrm{Ni}$ is taken from [24]. The dashed line is a guide to the eye.

\section{RESULTS AND DISCUSSION}

Due to the lack of suitable isotopes and the very low incoherent scattering cross section of aluminum, $D_{\mathrm{Al}}$ in $\mathrm{Al}-\mathrm{Ni}$ alloys cannot be measured. It is assumed to be equal to the Ni self-diffusion. This is supported by molecular-dynamic simulations for the concentrations considered [24]. More recent $a b$ initio $\mathrm{MD}$ simulations state that $D_{\mathrm{Al}}$ is about $1.5 \times D_{\mathrm{Ni}}$ [35]. We emphasize that deviations between $D_{\mathrm{Al}}$ and $D_{\mathrm{Ni}}$ have a minor influence on the discussion of cross correlations, since in Eq. (1) $D_{\mathrm{Al}}$ is multiplied by the low concentration of nickel. Even if $D_{\mathrm{Al}}$ were twice as high as $D_{\mathrm{Ni}}$ (i.e., comparable to pure aluminum [36,37]), the value of $S$ would differ by only $2 \%$ at a Ni concentration of 2 at. $\%$.

As Fig. 3 shows, $D_{\mathrm{AlNi}}$ at $1173 \mathrm{~K}$ is mostly constant for $\mathrm{Ni}$ concentrations between 1 and 14 at. $\% \mathrm{Ni}$ with a mean value of $(4.7 \pm 0.08) \times 10^{-9} \mathrm{~m}^{2} \mathrm{~s}^{-1}$. On ground, buoyancy may lead to disturbing convection, but density layering can have a stabilizing effect [38]. In contrast, under microgravity density-driven convection is greatly reduced, but persisting convection cannot be damped by density layering. Microgravity experiments, shown as stars in Fig. 3, agree with ground-based results within error bars. This is a strong indication that these diffusion data are undisturbed by convection.

While interdiffusion coefficients measured here are constant within error bars, Ni self-diffusion coefficients decrease with increasing $\mathrm{Ni}$ content. This behavior was also reported for concentrations above 10 at. \% Ni [24]. In Al-Ni with 20 at. $\% \mathrm{Ni}$, the interdiffusion (measured by long capillary) was reported to be faster than the Ni self-diffusion at $1380 \mathrm{~K}$ [25].

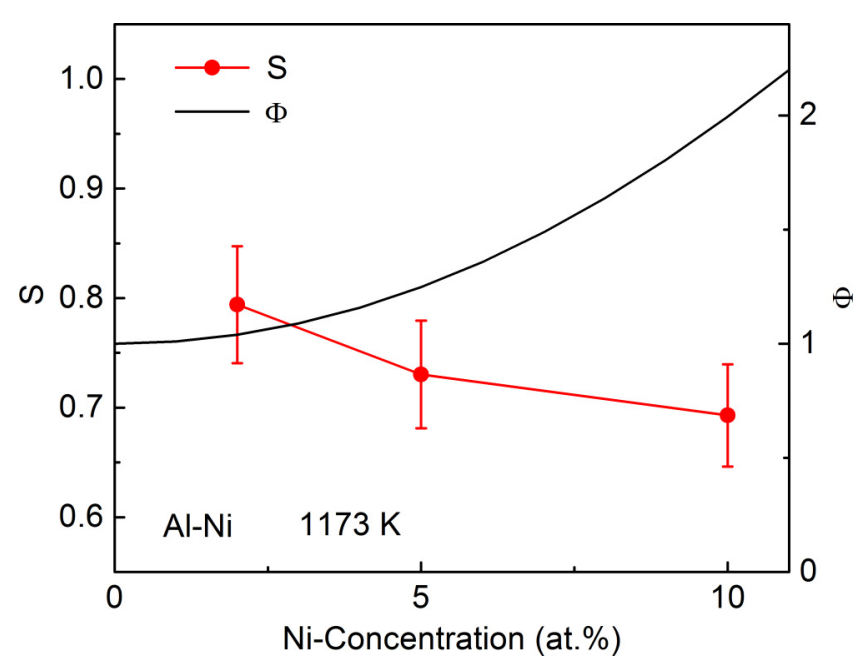

FIG. 4. Thermodynamic factor $\Phi$ derived from [42] and crosscorrelation term $S$ as calculated from experimental data as a function of $\mathrm{Ni}$ concentration.

To calculate the cross-correlation term $S$ via Eq. (1) using experimental data, the thermodynamic factor $\Phi$ is calculated from the second derivative of Gibbs free energy with respect to concentration. The thermodynamic description of Huang and Chang [39] results in a thermodynamic factor $\Phi<1$ for $\mathrm{Ni}$ concentrations below 7 at. \%. This does not fit to a miscible material with chemical short-range order like $\mathrm{Al}-\mathrm{Ni}$ [24,40,41]. In contrast, the thermodynamic factor obtained from the data given by Ansara et al. [42] is always greater than unity, as can be seen in Fig. 4. Therefore, the description by Ansara et al. is used in the following to obtain $\Phi$.

The determined cross-correlation term is below unity (see Fig. 4), which is typical for systems that exhibit a chemical short-range order. The error bars show the uncertainty originating from the measured diffusion coefficients. Estimating the uncertainty of $\Phi$ to be $10 \%$ adds $10 \%$ of $S$ to each error bar.

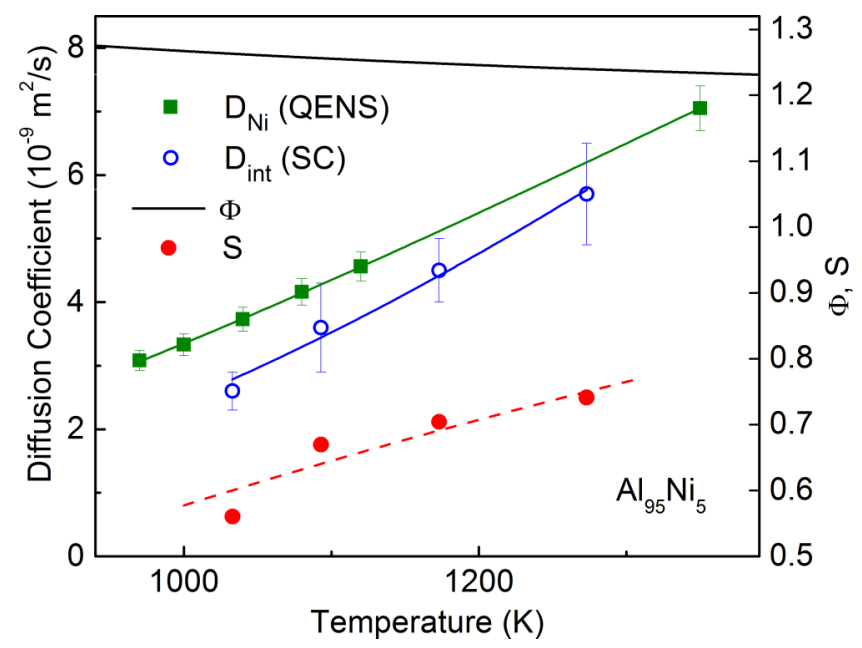

FIG. 5. Self- and interdiffusion coefficients of $\mathrm{Al}_{95} \mathrm{Ni}_{5}$ as a function of temperature. Solid lines are fits with the Arrhenius function. Also shown are the thermodynamic factor $\Phi$ calculated from [42] and the cross-correlation term $S$ resulting from experimental values. 
$S$ increases with decreasing $\mathrm{Ni}$ content. Although $S \rightarrow 1$ is expected for $c_{\mathrm{Ni}} \rightarrow 0$, the cross-correlation term is considerably below unity even at a concentration as low as 2 at. \% $\mathrm{Ni}$. At this concentration, it shows a value of $0.8 \pm 0.13$. For smaller concentrations of nickel, the Ni self-diffusion coefficient and the interdiffusion coefficient have to take the same value. This could be due to a drop of the Ni self-diffusion or an increase of interdiffusion or both.

In the temperature range $1033 \leqslant T \leqslant 1273 \mathrm{~K}$, the interdiffusion coefficient in $\mathrm{Al}_{95} \mathrm{Ni}_{5}$ is slightly lower than $D_{\mathrm{Ni}}$, as can be seen in Fig. 5. The temperature dependence of $\mathrm{Ni}$ self-diffusion and interdiffusion can be well described by the Arrhenius function $D=D_{0} \exp \left(-Q / k_{B} T\right)$ with an activation energy $Q$ and a prefactor $D_{0}$. The activation energy of Ni self-diffusion for $\mathrm{Al}_{95} \mathrm{Ni}_{5}$ is $Q=(247 \pm 1) \mathrm{meV}$. This compares well to activation energies found in pure $\mathrm{Al}[36,37]$ and in $\mathrm{Al}_{90} \mathrm{Ni}_{10}$ [24]. The measured data agree with activation energies determined by ab initio molecular-dynamics simulations of diffusion in Al-Ni melts [43]. Using the same procedure as described above, $S$ can be calculated. While the thermodynamic factor decreases slightly with temperature, the cross-correlation term increases. This means that the influence of cross correlations on interdiffusion decreases as the temperature increases.

\section{CONCLUSION}

In conclusion, the cross-correlation term $S$, calculated using the measured interdiffusion and Ni self-diffusion data and the thermodynamic factor taken from the literature, is below unity for Al-rich Al-Ni melts. The influence of cross correlations persists even at $\mathrm{Ni}$ concentrations as low as 2 at.\%. Hence, Darken's equation cannot be used in this case.

The interdiffusion coefficient is found to be mostly constant for Ni concentrations up to 14 at. \%. This result facilitates solidification simulations since the concentration dependence can be neglected. Whether this is also the case for other alloying metals will be the subject of future research.

\section{ACKNOWLEDGMENTS}

We thank Jörg Drescher, Michael Balter, and Christian Neumann for the implementation of the interdiffusion experiment on MAPHEUS-4. We gratefully acknowledge the allocation of beam time at the TOFTOF instrument operated by FRM II at the Heinz Maier-Leibnitz Zentrum (MLZ), Garching, Germany, and the help of Tobias Unruh during the neutron scattering experiment.
[1] E. L. Cussler, Diffusion: Mass Transfer in Fluid Liquid Systems, 3rd ed. (Cambridge University Press, Cambridge, 2009).

[2] L. Zhang, Y. Du, I. Steinbach, Q. Chen, and B. Huang, Acta Mater. 58, 3664 (2010).

[3] J. Campbell, Castings, 2nd ed. (Butterworth-Heinemann, Oxford, UK, 2003).

[4] J. Dantzig and C. Tucker, Modeling in Materials Processing (Cambridge University Press, Cambridge, 2001).

[5] I. Steinbach, Modell. Simul. Mater. Sci. 17, 073001 (2009).

[6] A. Borgenstam, L. Höglund, J. Agren, and A. Engström, J. Phase Equilib. 21, 269 (2000).

[7] G. Kasperovich, A. Meyer, and L. Ratke, Int. Foundry Res. 62, 8 (2010).

[8] T. Masaki, T. Fukazawa, S. Matsumoto, T. Itami, and S. Yoda, Meas. Sci. Technol. 16, 327 (2005).

[9] S. W. Basuki, A. Bartsch, F. Yang, K. Rätzke, A. Meyer, and F. Faupel, Phys. Rev. Lett. 113, 165901 (2014).

[10] F. Kargl, E. Sondermann, H. Weis, and A. Meyer, High Temp. High Press. 42, 3 (2013).

[11] M. Engelhardt, Ph.D. thesis, Rheinisch-Westfälischen Technischen Hochschule Aachen, 2014.

[12] H. Müller and G. Müller-Vogt, Cryst. Res. Technol. 38, 707 (2003).

[13] R. Roşu-Pflumm, W. Wendl, G. Müller-Vogt, S. Suzuki, K.-H. Kraatz, and G. Frohberg, Int. J. Heat Mass Transf. 52, 6042 (2009).

[14] Y. Malmejac and G. Frohberg, Mass Transport by Diffusion, in Fluid Sciences and Materials Science in Space: A European Perspective (Springer-Verlag, Berlin, 1987), pp. 159-190, Chap. V.

[15] M. Kassemi, S. Barsi, J. I. D. Alexander, and M. Banish, J. Cryst. Growth 276, 621 (2005).
[16] A. Griesche, B. Zhang, E. Solórzano, and F. Garcia-Moreno, Rev. Sci. Instrum. 81, 056104 (2010).

[17] B. Zhang, A. Griesche, and A. Meyer, Phys. Rev. Lett. 104, 035902 (2010).

[18] M. Siegl, F. Kargl, F. Scheuerpflug, J. Drescher, C. Neumann, M. Balter, M. Kolbe, M. Sperl, P. Yu, and A. Meyer Proceedings of the 21st ESA Symposium on European Rocket and Balloon Programmes and Related Research (ESA Communications, ESTEC, Noordwijk, The Netherlands, 2013)

[19] B. J. Yang, W. D. Huang, and R. W. Smith, J. Appl. Phys. 95, 7881 (2004).

[20] W. A. Arnold and D. Matthiesen, J. Electrochem. Soc. 142, 433 (1995).

[21] A. Meyer, EPJ Web Conf. 83, 01002 (2015).

[22] A. Meyer, Phys. Rev. B 81, 012102 (2010).

[23] A. Meyer and F. Kargl, Int. J. Microgravity Sci. Appl. 30, 30 (2013).

[24] S. K. Das, J. Horbach, M. M. Koza, S. M. Chatoth, and A. Meyer, Appl. Phys. Lett. 86, 011918 (2005).

[25] J. Horbach, S. K. Das, A. Griesche, M.-P. Macht, G. Frohberg, and A. Meyer, Phys. Rev. B 75, 174304 (2007).

[26] L. S. Darken, Trans. AIME 175, 184 (1948).

[27] P. Kuhn, J. Horbach, F. Kargl, A. Meyer, and T. Voigtmann, Phys. Rev. B 90, 024309 (2014).

[28] R. Sharma and K. Tankeshwar, J. Phys.: Condens. Matter 22, 455101 (2010).

[29] Y. Zhou and G. H. Miller, Phys. Rev. E 53, 1587 (1996).

[30] C. Neumann, E. Sondermann, F. Kargl, and A. Meyer, J. Phys. Conf. Ser. 327, 012052 (2011).

[31] E. Sondermann, C. Neumann, F. Kargl, and A. Meyer, High Temp. High Press. 42, 23 (2013). 
[32] J. Crank, The Mathematics of Diffusion, 2nd ed. (Oxford University Press, Oxford, 1999).

[33] J. Lee, S. Liu, H. Miyahara, and R. Trivedi, Metall. Mater. Trans. B 35, 909 (2004).

[34] J. P. Garandet, C. Barat, and T. Duffar, Int. J. Heat Mass Transf. 38, 2169 (1995).

[35] N. Jakse and A. Pasturel, J. Chem. Phys. 143, 084504 (2015).

[36] F. Kargl, H. Weis, T. Unruh, and A. Meyer, J. Phys. Conf. Ser. 340, 012077 (2012)

[37] F. Demmel, D. Szubrin, W.-C. Pilgrim, and C. Morkel, Phys. Rev. B 84, 014307 (2011).
[38] C. Barat and J. P. Garandet, Int. J. Heat Mass Transf. 39, 2177 (1996).

[39] W. Huang and Y. Chang, Intermetallics 6, 487 (1998).

[40] M. Maret, T. Pomme, A. Pasturel, and P. Chieux, Phys. Rev. B 42, 1598 (1990).

[41] A. Pasturel and N. Jakse, J. Phys.: Condens. Matter 27, 325104 (2015).

[42] I. Ansara, N. Dupin, H. L. Lukas, and B. Sundman, J. Alloys Compd. 247, 20 (1997).

[43] N. Jakse and A. Pasturel, Appl. Phys. Lett. 105, 131905 (2014). 